\title{
Pulmonary Artery Perforation: Rare reported Complication of Pericardiectomy for Chronic Constrictive Pericarditis recorded on Transesophageal Echocardiography
}

\author{
${ }^{1}$ Neeti Makhija, ${ }^{2}$ Jitin Narula, ${ }^{3}$ Sanjay Kumar, ${ }^{4}$ Sameer Taneja, ${ }^{5}$ Milind P Hote
}

\begin{abstract}
Major vessel injury is a possible complication during pericardiectomy for chronic constrictive pericarditis (CCP). We present a case of CCP, who while undergoing radical pericardiectomy incurred an iatrogenic injury of a major vessel with profuse bleeding. A rent in main pulmonary artery (MPA) was detected by the surgical team and simultaneously confirmed on transesophageal echocardiography (TEE), and a timely intervention was done. Importance of monitoring TEE in a patient undergoing pericardiectomy for CCP is highlighted.
\end{abstract}

Keywords: Constrictive pericarditis, Monitoring, Pericardiectomy, Pulmonary artery perforation, Transesophageal echocardiography.

How to cite this article: Makhija N, Narula J, Kumar S, Taneja S, Hote MP. Pulmonary Artery Perforation: Rare reported Complication of Pericardiectomy for Chronic Constrictive Pericarditis recorded on Transesophageal Echocardiography. J Perioper Echocardiogr 2016;4(1):17-18.

Source of support: Nil

Conflict of interest: None

\section{INTRODUCTION}

Major vessel injury is a rare but possible complication during pericardiectomy for chronic constrictive pericarditis (CCP). Dense calcified pericardial adhesions, radiation exposure, and previous surgery increase the risk of complications and warrant the use of elective cardiopulmonary bypass (CPB). We present the transesophageal echocardiography (TEE) image of iatrogenic main pulmonary artery (MPA) injury during radical pericardiectomy for CCP and also highlight the importance of monitoring TEE.

\footnotetext{
${ }^{1,5}$ Professor, ${ }^{2,3}$ Senior Resident, ${ }^{4}$ Trainee

${ }^{1-4}$ Department of Cardiac Anesthesia, All India Institute of Medical Sciences, New Delhi, India

${ }^{5}$ Department of Cardiothoracic and Vascular Surgery, All India Institute of Medical Sciences, New Delhi, India

Corresponding Author: Neeti Makhija, Professor, Department of Cardiac Anesthesia, All India Institute of Medical Sciences, New Delhi, India, Phone: +911126593423, e-mail: neetimakhija@hotmail.com
}

\section{CASE REPORT}

A 26-year-old male underwent pericardiectomy for CCP via median sternotomy at our institute. During pericardial dissection over the great vessels, dense adhesions with the underlying adventitia led to an accidental incision into a major vessel, resulting in profuse bleeding with hemodynamic compromise. A rent was noted by the surgical team on the anterior surface of the MPA. Transesophageal echocardiography was conducted using X7-2t Philips ultrasound probe and ultrasound machine (Phillips iE33 model, Bothell, WA, USA). A two-dimensional TEE with color flow Doppler in the mid-esophageal (ME) ascending aortic short-axis view at an angle of $44^{\circ}$ also revealed flow from the MPA into the pericardial cavity (Fig. 1, Video 1). This was managed aggressively with volume transfusion and use of vasoconstrictors till the time it was repaired using a side biting clamp. The subsequent surgery was then accomplished on femoro-femoral CPB.

\section{DISCUSSION}

During pericardiectomy, dissection of the thickened pericardium is carried superiorly onto the MPA trunk, as failure to relieve pericardial bands across it results in postoperative gradients and severe right ventricular

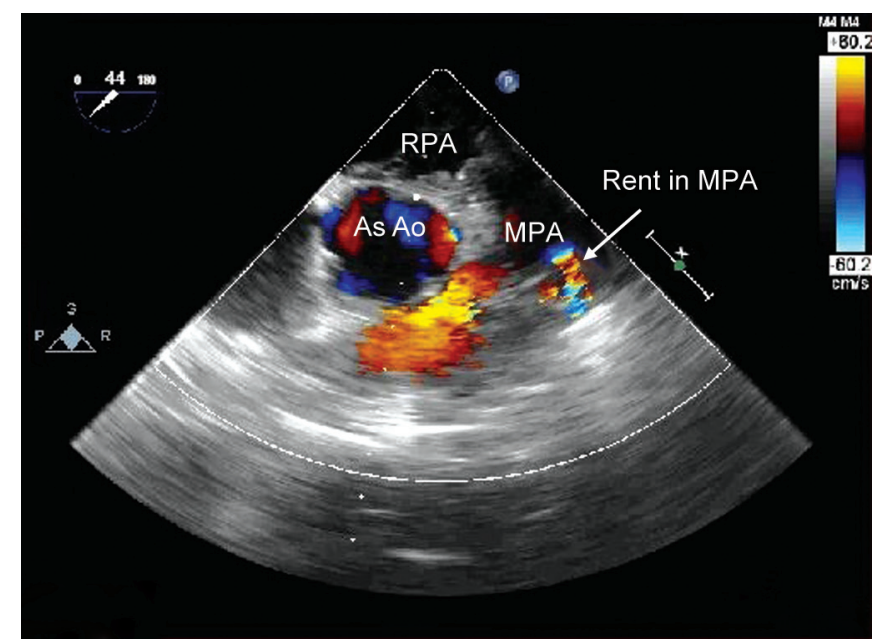

Fig. 1, Video 1: A two-dimensional mid-esophageal ascending aortic short-axis view at an angle of $44^{\circ}$; As Ao: Ascending aorta, MPA: Main pulmonary artery, RPA: Right pulmonary artery 
hypertension. ${ }^{1}$ Dissection of the aorta and pulmonary artery is performed first to avoid pulmonary edema. ${ }^{1}$ In our case, the absence of a dissection plane between the thickened pericardium and the adventitia of great vessels made the surgical dissection extremely challenging. Great vessel and cardiac chamber injuries are rare complications of pericardiectomy, which can result from dense calcific adhesions of the pericardium with the underlying epicardium, as was seen in our case. ${ }^{2,3}$ Earlier during the surgery, the major rent created during pulmonary artery dissection envisaged the subsequent use of $\mathrm{CPB}$, for the completion of the surgery, which was then uneventfully accomplished. Real-time intraoperative TEE evaluation of the great vessels in the ME ascending aortic short-axis view and the ME ascending aortic long-axis view thus provides valuable information on iatrogenic complications involving great vessel injury.

Transesophageal echocardiography is also an important monitoring tool to confirm the adequacy of resection during pericardiectomy for constrictive pericarditis. ${ }^{4}$ The resection is considered adequate when both ventricles, atrioventricular grooves, and the bases of great vessels are free of thickened pericardium. ${ }^{5}$ The pericardium in CCP is thickened, fibrosed, calcified, and echogenic and can be easily appreciated on TEE. Thus, any leftover thickened pericardium in the least accessible areas can be detected on TEE, thus guiding the surgeon to dissect pericardium in these areas. ${ }^{4}$ Also, the exaggerated respirophasic variations in transmitral blood flow velocities that occur in constrictive pericarditis are normalized following a complete pericardiectomy. ${ }^{6}$ After successful pericardiectomy, the improvement in ventricular filling can be appreciated on TEE. ${ }^{7}$ Although in some cases, pericardiectomy is associated with left ventricular dilatation and transient diastolic dysfunction, ${ }^{6}$ while performing pericardiectomy for constrictive pericarditis, epicardial coronary artery injury is a possibility. This can be easily detected as regional wall motion abnormality on TEE. ${ }^{4}$ In addition, with pericardiectomy the collapsibility of inferior venae cava improves, which can be confirmed on TEE. This can be correlated with concomitant fall in central venous pressures.

\section{CONCLUSION}

Major vascular injury during pericardiectomy for CCP is always a possibility and can be devastating if not timely and appropriately managed. Timely clamping of the involved vessel, till the time CPB can be initiated, helps in prevention of exsanguination. Transesophageal echocardiography can easily diagnose such complications and can facilitate early initiation of appropriate therapy. Also, TEE helps in confirmation of complete resection of the pericardium while performing pericardiectomy for constrictive pericarditis.

\section{REFERENCES}

1. Kirklin JW, Barrett-Boyes BG. Cardiac surgery: morphology, diagnostic criteria, natural history, techniques, results, and indications. 3rd ed. Vol. 2. New York (NY): Churchill Livingstone, Inc.; 2003. p. 1779-1798.

2. Bozbuga N, Erentug V, Eren E, Erdogan HB, Kirali K, Antal A, Akinci E, Yakut C. Pericardiectomy for chronic constrictive tuberculous pericarditis: Risks and predictors of survival. Tex Heart Inst J 2003;30(3):180-185.

3. Bertog SC, Thambidorai SK, Parakh K, Schoenhagen P, Ozduran V, Houghtaling PL, Lytle BW, Blackstone EH, Lauer MS, Klein AL. Constrictive pericarditis: Etiology and causespecific survival after pericardiectomy. J Am Coll Cardiol 2004 Apr;43(8):1445-1452.

4. Varadharajan R, Parida S, Badhe AS. TEE as a monitor during pericardiectomy for constrictive pericarditis. Indian J Thorac Cardiovasc Surg 2015 Jun;31(2):200.

5. Fitzpatrick DP, Wyso EM, Bosher LH, Richardson DW. Restoration of normal intracardiac pressures after extensive pericardiectomy for constrictive pericarditis. Circulation 1962 Mar;25(3):484-492.

6. Senni M, Redfield MM, Ling LH, Danielson GK, Tajik AJ, Oh JK. Left ventricular systolic and diastolic function after pericardiectomy in patients with constrictive pericarditis: Doppler echocardiographic findings and correlation with clinical status. J Am Coll Cardiol 1999 Apr;33(5):1182-1188.

7. Kasravi B, Ng D, Chandraratma PA. Continuous intraoperative transesophageal echocardiography during pericardiectomy for constrictive pericarditis revealing a dynamic change in chamber size. Echocardiography 2005 May;22(5):431-433. 